\title{
Acanthamoeba spp. como parásitos patógenos y oportunistas
}

\author{
Juan C. Castrillón y Lina P. Orozco
}

\section{Acanthamoeba spp. as opportunistic pathogens parasites}

Among free-living amoeba in nature, species of the genus Acanthamoeba have been associated with human disease. These amoeba are among the most abundant protozoa in nature due to its cosmopolitan distribution and are able to survive in a wide variety of habitats because its low demand for food and in harsh environments by forming structures known as cysts. However, ecological changes and incursion of its different habitats have made this organism can invade a host and live as parasites within him. That's why this type of protozoa are known as amphizoic organism, because human can be constituted as its host, causing infections in the central nervous system, disseminated infections in skin and lungs, and keratitis. Thus, since an increase in the number of cases of Acanthamoeba infections has occurred worldwide, these protozoa have become increasingly important as agents of human disease. This review summarizes what is known of this kind of free-living amoeba, focusing on the biology, ecology, pathogenesis, diagnosis, treatment and human defense mechanism against infection by the amoeba.

Key words: Acanthamoeba, pathogenesis, encephalitis, keratitis.

Palabras clave: Acanthamoeba, patogénesis, encephalitis, queratitis.

\section{Introducción}

A canthamoeba fue descrita por primera vez por Castellani en 1930, cuando reportó la presencia de una ameba en un cultivo de Cryptococcus pararoseus. Sin embargo, sólo en 1931 fue establecido su género, donde se ubican las amebas que presentan numerosas proyecciones espinosas, superficiales, conocidas como acantopodios ${ }^{1}$. El primer indicio de que Acanthamoeba podía causar enfermedades en humanos fue en 1958 durante los ensayos para la vacuna contra la polio. En cultivos celulares usados para preparar la vacuna, aparecieron placas que después de ser inoculadas en algunos modelos animales provocaron su muerte por encefalitis. Tiempo después se encontró que dichas placas fueron causadas por amebas pertenecientes al género Acanthamoeba. Estas observaciones llevaron a Culbertson a predecir el papel de las amebas de vida libre como agentes causantes de enfermedades en el hombre. Los primeros casos en los que Acanthamoeba fue establecida claramente como agente causal de enfermedades en humanos fueron reportados a partir de $1970^{2}$.

Desde que se conoció que diferentes amebas de vida libre pueden afectar el sistema nervioso central (SNC), el término encefalitis amebiana granulomatosa-EAG (en inglés GAE, granulomatous amebic encephalitis) ha sido usado para referirse a infecciones del SNC causadas por Acanthamoeba spp.; mientras que el término meningoencefalitis amebiana primaria- MEP (en inglés PAM, primary amebic meningoencephalitis) ha sido reservado para referirse únicamente a infecciones del SNC causadas por Naegleria fowleri. Los géneros Acanthamoeba y Naegleria se conocen como microorganismos anfizoicos debido a que tienen la capacidad de existir como amebas de vida libre y como parásitos patógenos y oportunistas ${ }^{3,4}$. Así, cada vez es más evidente que las amebas de vida libre pueden ser halladas como agentes causantes de infecciones en los seres humanos. En esta revisión se resume lo que se conoce de Acanthamoeba, un género de amebas de vida libre que ha venido ganando importancia debido al aumento de infecciones causadas en humanos en los últimos años.

\section{Biología y distribución}

Las especies de Acanthamoeba están entre los protozoos más frecuentes encontrados en la naturaleza. Están distribuidas por todo el mundo y han sido aisladas de tierra, polvo, aire, agua dulce natural y tratada, agua de mar, piscinas, aguas residuales, sedimentos, aire acondicionado, hospitales, lentes de contacto y cultivos celulares. También han sido aisladas de la vegetación; de animales incluyendo especies de peces, anfibios, reptiles y mamíferos; de la cavidad nasofaríngea de personas aparentemente sanas y de pacientes inmunodeficientes ${ }^{5}$.

El ciclo de vida de las especies de Acanthamoeba involucra dos estadios: una forma activa o trofozoito y una forma latente conocida como quiste (Figura 1). El trofozoito varía en tamaño de 25 a $40 \mu \mathrm{m}$ y se alimenta por fagocitosis de bacterias, algas y levaduras mediante seudópodos; aunque también puede vivir en forma axé-
Universidad de Antioquia, Medellín, Colombia. Facultad de Medicina. Sede de Investigación Universitaria Grupo Inmunovirología.

Los autores manifiestan que no existen, de manera directa o indirecta, conflictos de intereses, financieros, académicos ni personales para la publicación de este artículo.

No hubo financiamiento alguno.

Recibido: 10 de marzo de 2012 Aceptado: 4 de marzo de 2013

Correspondencia a: Juan Camilo Castrillón Betancur castricore@hotmail.com 
nica por medio de la captación de nutrientes disueltos en el ambiente, a través de un proceso de pinocitosis ${ }^{2}$. El quiste está compuesto de una doble pared, la exterior se conoce como ectocisto mientras que a la pared interna se le denomina endocisto (Figura 1). El tamaño del quiste

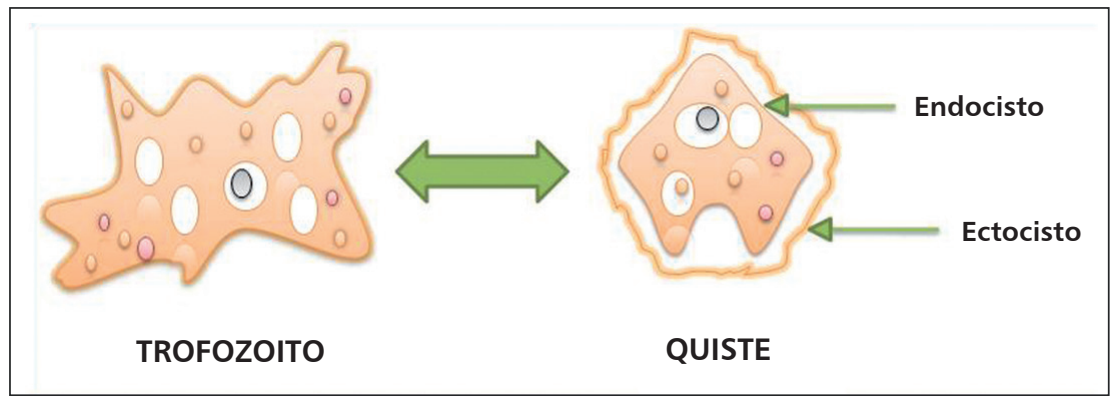

Figura 1. Estadios de Acanthamoeba. Bajo condiciones favorables, Acanthamoeba permanece en forma de trofozoito, mientras que en condiciones adversas se transforma en quiste.

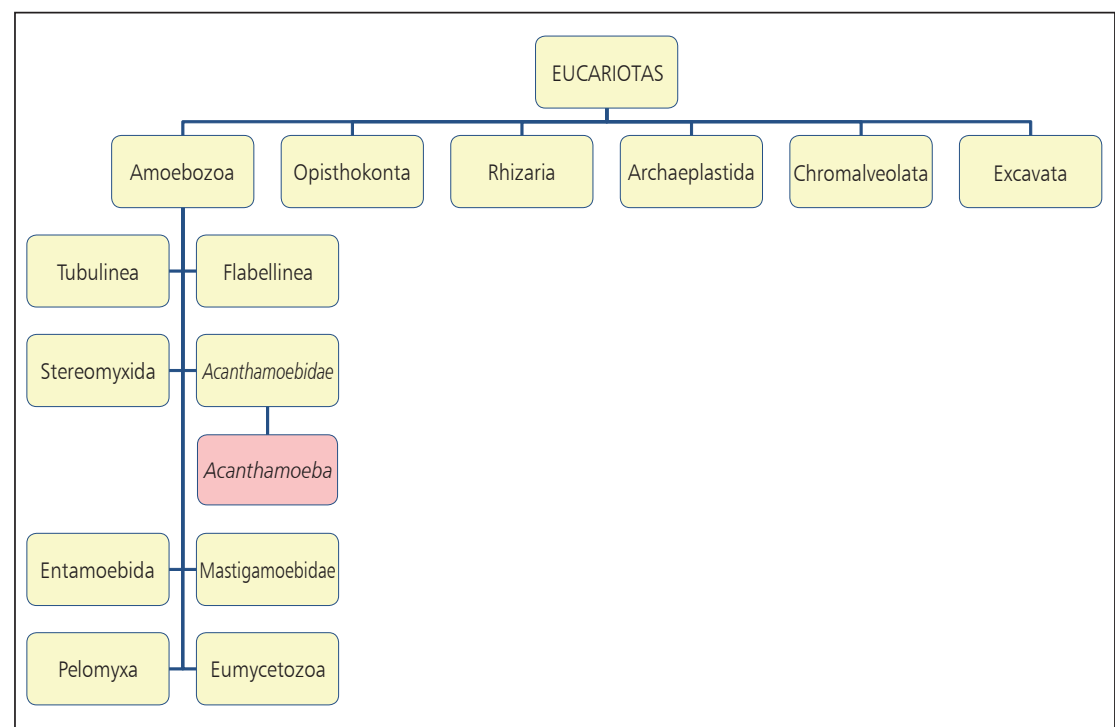

Figura 2. Clasificación del género Acanthamoeba dentro de los eucariotas. El género Acanthamoeba ha sido clasificado en el super grupo Amoebozoa, dentro de la categoría Acanthamoebidae.

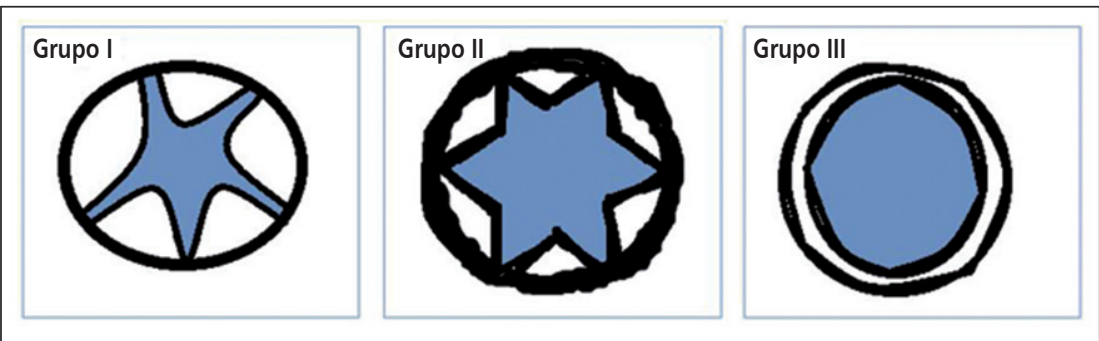

Figura 3. Clasificación de los quistes de Acanthamoeba en base a características morfológicas. El grupo I consiste de especies caracterizadas por grandes quistes y trofozoitos con endocistos en forma estrellada y ectocistos que pueden ser lisos o rugosos. El grupo II contiene amebas donde el ectocisto es rugoso y grueso, mientras que el endocisto puede ser poligonal, triangular, ovalado o redondeado. En el último grupo el ectocisto es delgado y ondulado mientras que el endocisto es generalmente redondeado. puede variar de 13 a $20 \mu \mathrm{m}$ de acuerdo a la especie. La formación del quiste ocurre bajo condiciones ambientales adversas como la falta de alimento, la desecación y los cambios en la temperatura y $\mathrm{pH}^{2}$.

\section{Taxonomía}

La clasificación taxonómica clásica en la que se dividió a los protozoos en cuatro grupos: Sarcodina, Mastigophora, Sporozoa e Infusoria, ha sido actualmente abandonada por una clasificación basada en nuevos enfoques morfológicos, rutas bioquímicas y filogenia molecular. El viejo sistema jerárquico que consistía en reino, filo, clase, subclase, superorden y orden, ha sido reemplazado por un nuevo vocabulario en el que los eucariotas han sido clasificados en seis super grupos (Figura 2). En tanto, el género Acanthamoeba ha sido clasificado dentro del super grupo Amoebozoa, dentro de la categoría Acanthamoebidae que se caracteriza por la presencia de proyecciones espinosas superficiales llamadas acantopodios y una forma latente (quiste) bajo condiciones ambientales adversas (Figura $1)^{6}$. Sin embargo, aún se ha mantenido para efectos de la identificación taxonómica la clasificación definida por Pussard y Pons en 1977, en la que el género Acanthamoe$b a$ se divide en tres grupos en base a las diferencias de tamaño y características morfológicas de los quistes ${ }^{7}$. El grupo I consiste en especies caracterizadas por grandes quistes y trofozoitos, con endocistos en forma estrellada y ectocistos que pueden ser lisos o rugosos, con un diámetro promedio del quiste $\geq 18 \mu \mathrm{m}$. El grupo II es el más grande, debido a que incluye a las amebas con una mayor distribución y las que han sido comúnmente aisladas. Este grupo contiene amebas con quistes $<18 \mu \mathrm{m}$, donde el ectocisto es rugoso, mientras que el endocisto puede ser poligonal, triangular, ovalado o redondeado. En el último grupo, el diámetro promedio de los quistes es $<18 \mu \mathrm{m}$ y el ectocisto es delgado y ondulado mientras que el endocisto es generalmente redondeado (Figura 3$)^{1}$. No obstante, la clasificación de Acanthamoeba basada en características morfológicas ha resultado poco fiable debido a que la morfología de las especies puede cambiar de acuerdo las condiciones de cultivo. Los criterios inmunológicos, bioquímicos y fisiológicos han sido aplicados para identificar las diferentes especies de Acanthamoeba; sin embargo, muchas especies comparten varias de estas características haciendo estos criterios de clasificación deficientes para su identificación ${ }^{8}$. Para evitar estos problemas, se han generado métodos de clasificación molecular, los cuales consisten en esquemas basados en secuencias del ARNr $18 \mathrm{~S}$, que generan linajes evolutivos denominados T1-T12 e involucran todos los aislados de Acanthamoeba encontrados hasta ahora ${ }^{9,10}$. Este enfoque ha sido fuertemente adoptado y hasta la fecha cinco nuevos genotipos han sido establecidos, denominados T13-T1 $17^{11-13}$. 


\section{Acanthamoeba spp como parásitos patógenos y oportunistas}

Las amebas del género Acanthamoeba tienen la capacidad de vivir como microorganismos de vida libre en la naturaleza y ocasionalmente invadir a un hospedero y vivir como parásitos dentro de él. Los tipos de infecciones más comunes producidas por Acanthamoeba spp. incluyen EAG, infecciones diseminadas (cutáneas y nasofaríngeas) y queratitis ${ }^{14}$. La ruta de invasión al SNC en los casos de EAG es probablemente a través de las vías respiratorias inferiores (Figura 4). Dentro de los síntomas clínicos característicos de esta patología, están las alteraciones en el estado mental, cambios en el comportamiento, convulsiones, cefalea, afasia, fiebre, rigidez de cuello, alteración de la visión, anorexia, náuseas y vómitos, ataxia, coma y muerte. La EAG está generalmente asociada a enfermos crónicos por tumores malignos, lupus, diabetes mellitus, falla renal, cirrosis, tuberculosis, entre otros. Entre los factores que predisponen su incidencia se incluyen el alcoholismo, el abuso de drogas, el embarazo, el tratamiento con corticoesteroides, la quimioterapia, la radioterapia y el trasplante de órganos. Sin embargo, la mayor susceptibilidad a la infección está asociada a condiciones que suprimen o debilitan el sistema inmunológico ${ }^{14}$.

Las especies del género Acanthamoeba también pueden infectar directamente la córnea causando queratitis (Figura 4). Esta infección es altamente resistente a terapias y frecuentemente resulta en una marcada deficiencia visual o incluso una pérdida total del ojo afectado. Cuando la infección es lo suficientemente grave, puede causar abrasión en la córnea ocasionando opacidad, iritis y a menudo escleritis. Los síntomas ocasionados incluyen enrojecimiento, epífora, dolor intenso, fotofobia y edema. El uso de lentes de contacto o un trauma en la córnea son los principales factores de riesgo asociados a esta infección ${ }^{15}$.

En algunos pacientes se han encontrado lesiones en la piel que se caracterizan por la presencia de nódulos duros eritematosos y úlceras dolorosas en el tronco o extremidades $^{16}$. La acantamebiasis cutánea, a menudo es un reflejo de la enfermedad diseminada; es una infección cada vez más reconocida desde la aparición del síndrome de inmunodeficiencia adquirida (SIDA) y el uso de fármacos inmunosupresores, generalmente en pacientes receptores de trasplantes. La enfermedad augura un mal pronóstico y es fatal si la infección afecta al $\mathrm{SNC}^{17}$. Además, se han encontrado trofozoitos y quistes en tejido pulmonar produciendo lesiones consistentes con neumonitis. Este tipo de infecciones, junto con la bronquiolitis, siguen siendo una importante causa de mortalidad durante los primeros años después de un trasplante de pulmón ${ }^{18}$.

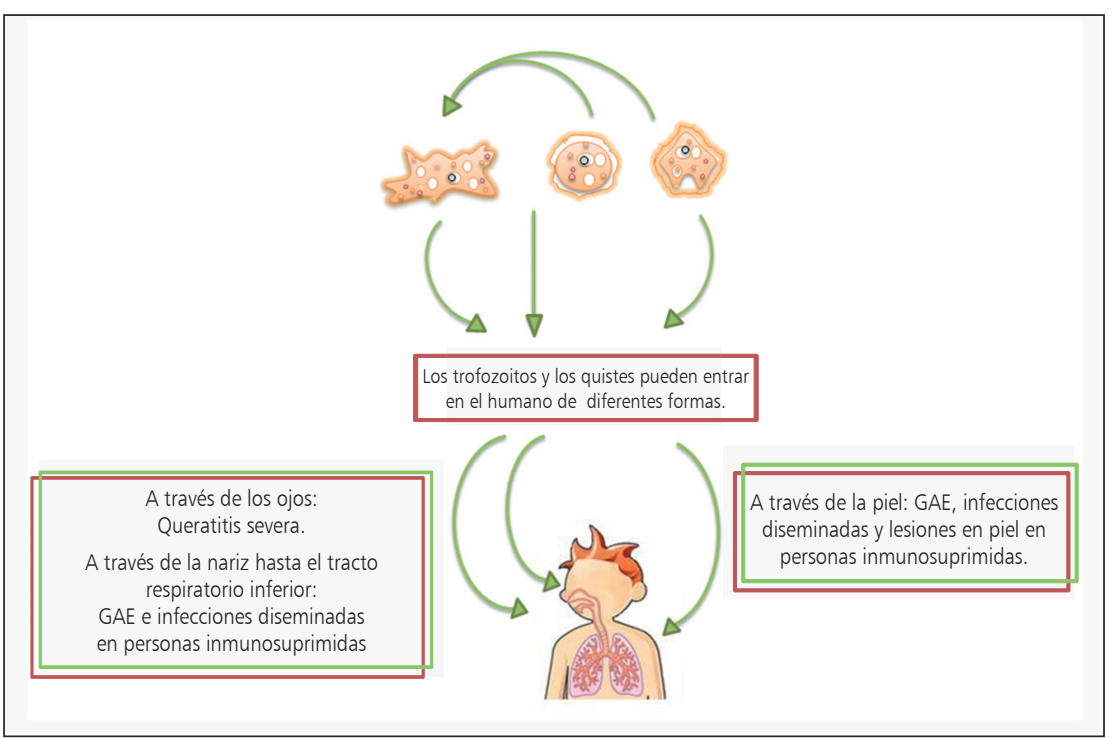

Figura 4. Rutas de entrada de Acanthamoeba a su hospedero. Acanthamoeba puede entrar a través de los ojos y causar queratitis grave en personas sanas; a través de las fosas nasales o de la piel ulcerada e invadir el SNC y causar EAG o lesiones de la piel en personas con sistemas inmunes comprometidos.

\section{Patogenia de la infección por Acanthamoeba}

La patogénesis de la acantamebiasis es un proceso complejo que involucra varios determinantes trabajando en concierto para producir la enfermedad. El primer paso crítico es su adhesión a la superficie de los tejidos ${ }^{19}$ a través de la expresión de una proteína transmembranal de aproximadamente $400 \mathrm{kDa}$ conocida como proteína de unión a manosa-PUM (en inglés: mannose-binding protein-MBP), que se une a glicoproteínas que contienen manosa expresadas en las células blanco ${ }^{20,21}$. La PUM contiene en su porción extracelular repeticiones ricas en cisteína, las cuales también podrían inducir la unión de protozoos individuales y llevar a la formación de agregados ${ }^{22}$. Además de esta adhesina, otra podría estar participando en la patogénesis de Acanthomoeba: la proteína de unión a laminina, uno de los principales constituyentes de la matriz extracelular ${ }^{23}$. Subsecuente a la adhesión, la ameba produce diferentes enzimas hidrolíticas como proteasas y fosfolipasas, que trabajan en conjunto para producir un potente efecto citopático que involucra el incremento en la concentración de $\mathrm{Ca}^{++}$, cambios en la estructura del cito-esqueleto y en la morfología celular, aumento en la permeabilidad de la membrana celular y mitocondrial, degradación de la matriz extracelular y finalmente, la muerte de la célula. Se conoce también que este microorganismo expresa ecto-ATPasas en su membrana que hidrolizan el ATP en ADP, el que puede unirse a receptores celulares causando un incremento en la concentración de $\mathrm{Ca}^{++}$, lo que activa diferentes cascadas de señalización que llevan a la muerte 


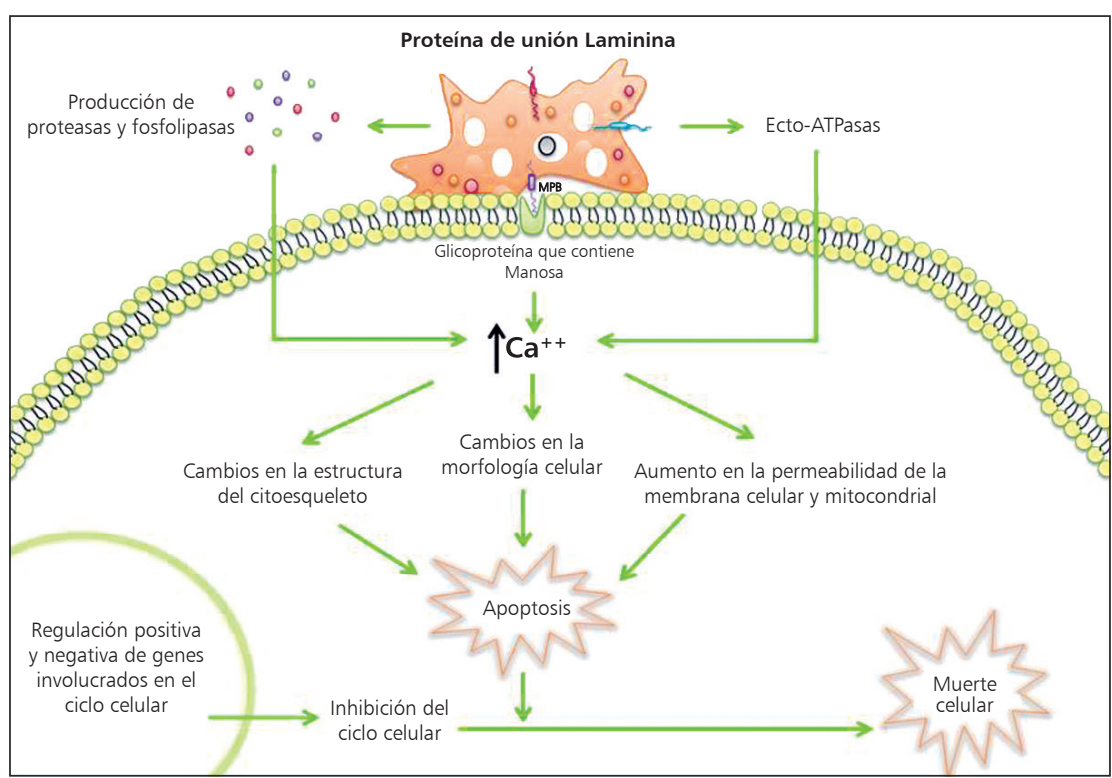

Figura 5. Patogénesis de Acanthamoeba. La patogénesis de Acanthamoeba involucra la adhesión a la superficie de los tejidos a través de la expresión de la proteína MBP. Y la producción de diferentes enzimas hidrolíticas que trabajan en conjunto para producir un potente efecto citopático que involucra la degradación de la matriz extracelular y la muerte celular.

de la célula por apoptosis ${ }^{24}$. Otro aspecto importante en la virulencia de Acanthomoeba parece ser la capacidad de fagocitar la célula blanco; un proceso que requiere la inducción de re-arreglos en el cito-esqueleto y la formación de amoebastomas en la superficie celular. Esto ha sido reportado en cultivos de células epiteliales de córnea con Acanthomoeba, donde se observa la formación de los amoebastomas después de cierto tiempo de incubación y gracias a la inhibición de la actividad fagocítica de la ameba cuando se utilizan inhibidores de la polimerización de actina como la citocalasina $\mathrm{D}^{25}$. Este parásito también ejerce su acción patogénica bloqueando genes importantes en el proceso de división celular, lo que induce la muerte de la célula y evita la regeneración tisular ${ }^{23}$. En la Figura 5. se muestra un esquema resumido de la patogénesis de Acanthomoeba.

\section{Aspectos inmunológicos}

La protección frente a una infección por este patógeno puede involucrar tanto la inmunidad innata como la adaptativa. Una de las principales razones por las que este tipo de patógeno no infecta a individuos totalmente saludables es su capacidad de activar la vía del complemento. Es muy probable que el complemento provea una de las primeras líneas de defensa natural frente a una infección por Acanthamoeba. Los hallazgos en los que A. culbertsoni es neutralizada al ponerse en contacto con suero humano deficiente en $\mathrm{C} 2$, indican la activación de la ruta alternativa del complemento en presencia de $A$. culbertsoni dilucidando la importancia de esta ruta como un mecanismo de defensa natural ante una infección inicial por $A$. culbertsoni en pacientes inmunocompetentes $^{26}$. De la misma forma, recientemente Pumidonming y cols., confirmaron las propiedades amebolíticas del suero humano a través de la activación del complemento vía la ruta alternativa ${ }^{27}$. También se ha reportado que la respuesta más temprana del hospedero consiste en un reclutamiento de neutrófilos en el lugar de la infección. La presencia temprana de neutrófilos activados podría evitar la diseminación e invasión de Acanthamoeba además de limitar la oportunidad de los trofozoitos de infiltrar la córnea y eventualmente evitar su enquistamiento ${ }^{28}$. Los estudios han demostrado que el mecanismo utilizado para controlar la infección por parte de los neutrófilos involucra la producción de mieloperoxidasas ${ }^{29}$.

Además de los neutrófilos, los macrófagos son también una de las primeras células inflamatorias que migran al lugar de la infección. Las investigaciones sugieren que los macrófagos pueden eliminar quistes y trofozoitos de Acanthamoeba por medio de fagocitosis. Sin embargo, estas células de la inmunidad innata, sólo pueden responder eficazmente a la infección en presencia de algunos anticuerpos y citoquinas que permitan su activación ${ }^{29,30}$. Algunos de estos factores inflamatorios como IL-8, FNT- $\alpha$ e IFN- $\beta$ son inducidos a través de la activación del receptor tipo Toll 4 (TLR4) en respuesta a una queratitis causada por Acanthamoeba. Estos receptores juegan un papel muy importante en la inmunidad innata a través de la detección de patógenos como bacterias, hongos y virus, debido a que inducen una señal que permite la regulación de la síntesis de citoquinas inflamatorias necesarias para la regulación de la función de células del sistema inmune ${ }^{31}$.

Algunos pacientes y modelos animales con queratitis, han sido susceptibles a una reinfección por Acanthamoeba sugiriendo que la memoria inmunológica no se desarrolla en respuesta a esta infección. Estas observaciones indican que la respuesta inmune adaptativa no es adecuadamente activada en reacción a infecciones oculares por Acantha$m o e b a^{32}$. La ausencia de células presentadoras de antígeno residentes en la córnea es probablemente la causa de la no activación del sistema inmune adaptativo a una infección en la córnea ${ }^{33}$. Sin embargo, MCclellan y cols., encontraron que los quistes de $A$. castellanii podían generar una producción de $\mathrm{IgG}$ específicos y una proliferación de linfocitos $\mathrm{T}$, indicando que estos quistes pueden ser reconocidos por el sistema inmune ${ }^{34}$.

El papel de los anticuerpos en la infección por Acanthamoeba todavía no se ha logrado esclarecer totalmente, pero se ha demostrado que pueden prevenir su adhesión a las células blanco, inhibir su movilidad y neutralizar algunos de sus factores citotóxicos. El epitelio de la mucosa ocular está protegido por una variedad de componentes 
presentes en las lágrimas que incluyen lactoferrina, lisozimas y lactoperoxidasas, además de IgA, IgG e IgM. IgA es la inmunoglobulina más abundante en este tipo de fluidos y se ha encontrado que puede prevenir el establecimiento de una infección en córnea por Acanthamoeba, inhibiendo la adhesión del parásito al epitelio corneal, pero no afecta su viabilidad una vez se ha iniciado la infección ${ }^{35}$.

En los últimos años, se ha reportado que las células microgliales, una población de macrófagos residentes en el cerebro, pueden ejercer una actividad amebicida. Esto fue demostrado en un co-cultivo de células microgliales obtenidas de ratón con $A$. castellanii, en el que las células microgliales tenían la capacidad de fagocitar y lisar las amebas, además de inducir la producción de citoquinas como IL- $1 \alpha$, IL- $1 \beta$ y FNT- $\alpha$ que actúan como mediadores de la inflamación local al estimular la atracción y activación de neutrófilos y monocitos hacia las zonas de infección ${ }^{36}$. Sin embargo, estudios recientes indican que $A$. culbertsoni secreta serín peptidasas que degradan citoquinas producidas por células microgliales de ratón y además pueden inducir la apoptosis de dichas células ${ }^{37}$.

\section{Metabolismo}

Con el fin de proporcionar una plataforma para el estudio molecular de este patógeno oportunista de importancia clínica, responsable de diversas enfermedades en el hombre, diversos estudios han permitido dilucidar la capacidad de Acanthamoeba para sobrevivir en un amplio rango de ambientes, incluyendo el hombre como hospedero accidental ${ }^{21,38}$. Los datos generados predicen que Acanthamoeba exhibe una gran capacidad biosintética de aminoácidos, co-factores y nucleótidos, moléculas orgánicas esenciales para su crecimiento. Los resultados sugieren también que Acanthamoeba puede incrementar la diversidad de sus fuentes de alimento, ya que tiene la capacidad de sintetizar enzimas como la alginatoliasa que le proporciona acceso a las bacterias a través de la matriz extracelular que compone la biopelícula; enzimas como la polihidroxibutirato despolimerasa que facilita la utilización del PHA presente en el interior de las bacterias como fuente de alimento; enzimas para la síntesis y degradación de la celulosa y la enzima sintasa trehalosa-6-fosfato que juega un papel importante en la adaptación al estrés ${ }^{39}$.

\section{Diagnóstico de la infección por Acanthamoeba}

El diagnóstico de una infección por Acanthamoeba ha incluido, hasta ahora, diversas técnicas donde el método de cultivo in vitro es todavía usado y en algunos casos el único disponible para confirmar la infección. Muestras de líquido cefalorraquídeo, tejido cerebral, material cutáneo o raspado de córnea pueden ser inoculados en medio de crecimiento para amebas ${ }^{2}$. Acanthamoeba puede ser cultivada en agar no nutritivo o agar con una baja concentración de nutrientes (peptona $0,05 \%$, extracto de levadura $0,05 \%$ y glucosa $0,1 \%$ ) en presencia de bacterias. Las bacterias incluyen especies no mucoides como Klebsiella pneumoniae, Enterobacter spp. (Enterobacter aerogenes y Enterobacter cloacae) y Escherichia coli debido a que la presencia de una cápsula mucoide rodeando la bacteria puede impedir la fagocitosis ${ }^{40}$. La presencia de la ameba puede examinarse en la superficie del agar a través de un microscopio. En el caso de que se requiera aislar Acanthamoeba del ambiente o de muestras clínicas, es necesario usar un medio no nutritivo ya que la presencia de glucosa fomentaría el crecimiento de bacterias no deseadas que inhiban la proliferación de la ameba ${ }^{3}$.

También es posible realizar un diagnóstico histológico a partir de biopsias de piel, cerebro o córnea, utilizando diferentes métodos de tinción. La tinción hematoxilinaeosina no es diferencial pero macrófagos y células del sistema inmune se distinguen de la ameba debido a diferencias en la morfología nuclear. La estructura nuclear de Acanthamoeba se caracteriza por un pronunciado cariosoma rodeado de un halo. Sin embargo, otros métodos de tinción han resultado ser muy útiles para la identificación de quistes en secciones de tejidos. La tinción de PAS (del inglés periodic acid-Schiff) colorea de rojo la pared del quiste, mientras que la tinción de Gomori Grocott- (metenamina-plata) tiñe el quiste de negro. Además, la tinción con calflúor blanco, un material que se une a celulosa de los quistes y emite fluorescencia cuando es excitado, es usado para identificar quistes en tejido cerebral ${ }^{15}$. Estos métodos de tinción revelan generalmente las amebas localizadas en espacios perivasculares e invadiendo vasos sanguíneos. El uso de inmunofluorescencia empleando anticuerpos específicos contra especies de Acanthamoeba ha sido usado para detectar amebas en biopsias de tejidos. También se ha empleado la observación directa de la córnea a través de microscopia confocal para el diagnóstico de queratitis ${ }^{41,42}$.

Además de los métodos de tinción, el uso de la técnica de reacción de polimerasa en cadena-RPC (en inglés polymerase chain reaction- $P C R$ ) y el análisis de la variación de las secuencias de ADN a través de la técnica de polimorfismos de longitud de fragmentos de restricción-PLFR (en inglés restriction fragment length polymorphism-RFLP), han demostrado ser métodos efectivos y sensibles ${ }^{43-45}$.

\section{Epidemiología y control de infecciones causadas por Acanthamoeba}

El número de infecciones causadas por Acanthamoe$b a$ es relativamente pequeño a pesar de la distribución mundial del parásito, ya que es casi que imposible evitar 
su contacto. Desde 1960, cuando se reportó el primer caso, hasta el año 2000, el número de casos reportados de individuos con EAG fue de aproximadamente $200^{46}$. Aproximadamente 17 especies de Acanthamoeba han sido descritas pero sólo cinco han sido asociadas a infecciones sistémicas en el hombre: A. polyphaga, A. castellanii, $A$. culbertsoni, $A$. hatchetti y $A$. healyi ${ }^{46}$. Además de casos en humanos, las infecciones sistémicas por Acanthamoeba han sido reportadas en ovejas, perros, monos y caballos $^{47,48}$.

Por otra parte, la queratitis amebiana ha sido reportada en más de 3.000 individuos hasta el año 2000, siendo las causas más comunes de esta infección el uso inapropiado de lentes de contacto por mujeres y un trauma corneal en hombres mayores a 50 años. Cabe resaltar que los individuos que desarrollan queratitis son saludables y no presentan tipo alguno de inmunodeficiencia ${ }^{44}$.

El desarrollo y uso extensivo de lentes de contacto desde 1980 ha resultado en la aparición frecuente de casos de queratitis por Acanthamoeba. Antes de ese período, su ocurrencia era rara y estaba limitada a un trauma corneal accidental. Con el uso generalizado de lentes de contacto y la falta de cuidados para su mantenimiento, la queratitis amebiana ha alcanzado proporciones epidémicas con casos reportados en todo el mundo ${ }^{44}$. El problema se agravó por la falta de agentes antimicrobianos efectivos para su tratamiento. Sin embargo, en la actualidad el personal médico tiene más conocimiento de esta infección, aunque sigue siendo el diagnóstico tardío un condicionante de la gravedad de los cuadros de queratitis. Está disponible un tratamiento eficaz, aunque esta eficacia es relativa y dependerá del grado de avance de la infección y, afortunadamente, los usuarios de lentes de contacto están mejor informados de los riesgos de no cumplimiento de las recomendaciones para el cuidado de las lentes, ayudando a prevenir una de las principales causas de queratitis.

Evitar el contacto con este microorganismo es imposible debido a su ubicuidad; sin embargo, es posible tener algunas sencillas precauciones para minimizar el contacto con este parásito. En primer lugar, los individuos inmunodeficientes deben evitar la exposición al polvo y a partículas del suelo que pueden llevar quistes al sistema respiratorio, evitar piscinas o aguas termales que no estén tratadas correctamente y la exposición al ambiente de heridas abiertas. En el caso de queratitis, si se usan lentes de contacto, lo ideal es mantenerlos limpios y evitar nadar con ellos.

\section{Terapéutica}

Para el tratamiento de infecciones sistémicas se han usado compuestos como ketoconazol, fluconazol, itraconazol, pentamidina isetionato, cotrimoxazol, sulfadiazina, y 5-fluorocitosina (flucitosina); en forma individual o combinada, con diferentes grados de buen éxito. Infecciones cutáneas han sido tratadas con aplicaciones tópicas de gluconato de clorhexidina y ketoconazol ${ }^{49}$. Algunos regímenes de tratamiento exitosos han incluido cotrimoxazol, rifampicina y ketoconazol en dos pacientes pediátricos con infecciones del $\mathrm{SNC}^{50}$; cotrimoxazol, flucitosina y sulfadiazina en un paciente pediátrico con infección del $\mathrm{SNC}^{51}$; pentamidina intravenosa, clorhexidina y ketoconazol tópicas en un paciente con trasplante renal con infección diseminada ${ }^{52}$; sulfadiazina, y fluconazol en un paciente con infección por VIH/SIDA con EAG ${ }^{53}$. A pesar de esta cadena de eventos exitosos, la eficacia de un fármaco o combinación de fármacos en detener una infección por Acanthamoeba está determinada por un número de factores, incluyendo el estado inmune del hospedero, la progresión de la enfermedad y el momento en que se inicia la intervención del medicamento, la dosis infecciosa de las amebas, el perfil de susceptibilidad al fármaco y la virulencia de la cepa particular que causa la infección.

El tratamiento para la EAG es muy inseguro debido a la falta de síntomas claros, la falta de una buena prueba diagnóstica fiable, y el hecho de que el diagnóstico se hace a menudo post-mortem. Sin embargo, algunos pacientes con EAG han sido exitosamente tratados con sulfadiazina y fluconazol, además de que diversos fármacos han sido utilizados con variables grados de éxito y en algunos casos el tratamiento es interrumpido debido a efectos secundarios indeseables de los medicamentos ${ }^{53,54}$. Una combinación de factores, entre ellos el diagnóstico tardío, la eficacia sub-óptima de la terapia antiparasitaria y problemas inherentes al hospedero (inmunodeficiencias) hacen que la perspectiva de un tratamiento exitoso para la EAG sea pobre ${ }^{3}$.

Los trofozoítos de Acanthamoeba son sensibles a los agentes quimioterapéuticos más comunes (antimicrobianos, antisépticos, antifúngicos, antiprotozoarios, antivirales y agentes antineoplásicos). Sin embargo, la persistencia de la infección está relacionada con la presencia de quistes contra los pocos agentes químicos que tienen algún efecto ${ }^{55}$. Las diamidinas y biguanidas son actualmente los cisticidas más eficaces in vitro y su uso está respaldado por una cadena de casos exitosos (Tabla 1). Dentro de las biguanidas usadas para el tratamiento de la queratitis amébica, está el polihexametileno biguanida (PHMB) que se utiliza como limpiador de piscinas y el gluconato de clorhexidina que es un componente de jabones germicidas. Las biguanidas interactúan con la membrana citoplasmática celular aumentando su permeabilidad lo que resulta en la pérdida de componentes celulares; además, se une al ADN y altera su transcripción ${ }^{42}$. Los estudios in vitro han demostrado que estos dos agentes tienen la mejor y más constante actividad amebicida y cisticida 
Tabla 1. Regímenes de tratamiento y resultados para infecciones por Acanthamoeba

\begin{tabular}{|lcc|}
\hline Tratamiento & Resultado & Referencia \\
PHMB + clorhexidina & Exitoso & 57 \\
$\begin{array}{l}\text { Gluconato de chlorhexidina }+ \\
\text { neosporina }\end{array}$ & Beneficioso & 58 \\
PHMB + propamidina & Efectivo & 59 \\
$\begin{array}{l}\text { Pentamidina + itraconazol }+ \\
\text { gluconato de chlorhexidina }\end{array}$ & Exitoso & 60 \\
$\begin{array}{l}\text { Clorhexidina } \\
\text { Clorhexidina + propamidina }\end{array}$ & Exitoso & 61 \\
\hline PHMB: polihexametileno biguanida. & & 62 \\
\hline
\end{tabular}

entre los fármacos estudiados hasta la fecha, por lo que son usadas como tratamiento de primera línea contra la queratitis amébica ${ }^{56}$. Dentro de las diamidinas disponibles están isetionato de propamidina y hexamidina. Estas dos afectan la permeabilidad y desnaturalizan enzimas y proteínas en el citoplasma. Sin embargo, a diferencia de las biguanidas, el tratamiento prolongado con diamidinas puede llegar a ser tóxico ${ }^{42}$.

\section{Conclusiones}

A pesar de que la mayoría de los casos de encefalitis amebiana reportados en la literatura médica lo han sido en países en los que están disponibles procedimientos especializados para la identificación de estos casos, su alta mortalidad es, en gran parte, la consecuencia de la falta de médicos familiarizados con este tipo de enfermedades amebianas, retraso en el diagnóstico y la falta de un tratamiento óptimo.

El progreso humano ha facilitado la presencia de Acanthamoeba en la población, además de que una serie de factores pueden explicar el aumento en la incidencia de infecciones causadas por este parásito: la popularidad de las lentes de contacto, la disponibilidad de los medicamentos inmunosupresores que permiten el trasplante de órganos, el gran número de personas infectadas por el VIH y el aumento de pacientes sometidos a quimioterapia para el tratamiento del cáncer.

Agradecimientos. A la universidad de Antioquia por aportarnos todo el conocimiento que hasta ahora nos hace buenos profesionales.

\section{Resumen}

Entre las amebas de vida libre (AVL) que existen en la naturaleza, las especies pertenecientes al género Acanthamoeba han sido asociadas a enfermedades en humanos. Las AVL están entre los protozoos más abundantes en la naturaleza debido a su distribución cosmopolita y a que pueden sobrevivir en una amplia variedad de hábitats, incluyendo ambientes inhóspitos, gracias a su poca demanda de alimento y a que puede formar estructuras conocidas como quistes que las hacen resistentes. Los cambios ecológicos y la incursión de sus diferentes hábitats, han hecho que puedan invadir un hospedero y vivir como parásitos dentro de él. Por esto, este protozoo se conoce como un microorganismo anfizoico, ya que el hombre puede llegar a constituirse como su hospedero, causando infecciones en el sistema nervioso central, infecciones diseminadas en piel y pulmones, y queratitis. Es así como desde el incremento en el número de infecciones reportadas en el mundo por Acanthamoeba, estos protozoos se han convertido en importantes agentes de enfermedades en el hombre. En esta revisión se resume lo que se conoce de este género de AVL, enfocándose en su biología, patogénesis y los mecanismos de defensa humano frente a la infección por Acanthamoeba.

\section{Referencias bibliográficas}

1.- Visvesvara G S. Classification of Acanthamoeba. Rev Infect Dis 1991; 13 Suppl 5: S369-72.

2.- Marciano-Cabral F, Cabral G. Acanthamoeba spp. as agents of disease in humans. Clin Microbiol Rev 2003; 16: 273-307.

3.- Visvesvara G S, Moura H, Schuster F L. Pathogenic and opportunistic free-living amoebae: Acanthamoeba spp., Balamuthia mandrillaris, Naegleria fowleri, and Sappinia diploidea. FEMS Immunol Med Microbiol 2007; 50: 1-26.

4.- Oddo BD. Infections caused by free-living amebas. Historical commentaries, taxonomy and nomenclature, protozoology and clinicopathologic features. Rev Chilena Infectol 2006; 23: 200-14.

5.- De Jonckheere J F. Ecology of Acanthamoeba. Rev Infect Dis 1991; 13 Suppl 5: S385-7.

6.- Adl S M, Simpson A G, Farmer M A, Andersen R A, Anderson O R, Barta J R, et al. The new higher level classification of eukaryotes with emphasis on the taxonomy of protists. J Eukaryot Microbiol 2005; 52: 399-451.

7.- Pussard M, Pons R. Morphologie de la paroi kystique et taxonomie du genre Acanthamoeba (Protozoa, Amoebida). Protistologica 1977; 13: 557-98.

8.- Johnson A M, Fielke R, Christy P E,
Robinson B, Baverstock P R. Small subunit ribosomal RNA evolution in the genus Acanthamoeba. J Gen Microbiol 1990; 136: 1689-98.

9.- Stothard D R, Schroeder-Diedrich J M, Awwad M H, Gast R J, Ledee D R, RodríguezZaragoza $\mathrm{S}$, et al. The evolutionary history of the genus Acanthamoeba and the identification of eight new $18 \mathrm{~S}$ rRNA gene sequence types. J Eukaryot Microbiol 1998; 45: 45-54.

10.- Gast R J, Ledee D R, Fuerst P A, Byers T J. Subgenus systematics of Acanthamoeba: four nuclear 18S rDNA sequence types. J Eukaryot Microbiol 1996; 43: 498-504.

11.- Hewett M K, Robinson B S, Monis P T, Saint C P. Identification of a new Acanthamoeba 
18S rRNA gene sequence type, corresponding to the species Acanthamoeba jacobsi Sawyer, Nerad and Visvesvara, 1992 (Lobosea: Acanthamoebidae). Acta Protozool 2003; 42: 325-9.

12.- Corsaro D, Venditti D. Phylogenetic evidence for a new genotype of Acanthamoeba (Amoebozoa, Acanthamoebida). Parasitol Res 2010; 107: 233-8.

13.- Nuprasert W, Putaporntip C, Pariyakanok L, Jongwutiwes S. Identification of a novel $\mathrm{t} 17$ genotype of Acanthamoeba from environmental isolates and t10 genotype causing keratitis in Thailand. J Clin Microbiol 2010; 48: 4636-40.

14.- Martínez A J. Infection of the central nervous system due to Acanthamoeba. Rev Infect Dis 1991; 13 Suppl 5: S399-402.

15.- Ma P, Visvesvara G S, Martínez A J, Theodore F H, Daggett P M, Sawyer T K. Naegleria and Acanthamoeba infections: review. Rev Infect Dis 1990; 12: 490-513.

16.- Martínez A J, Visvesvara G S. Free-living, amphizoic and opportunistic amebas. Brain Pathol 1997; 7: 583-98.

17.- Paltiel M, Powell E, Lynch J, Baranowski B, Martins C. Disseminated cutaneous acanthamebiasis: a case report and review of the literature. Cutis 2004; 73: 241-8.

18.- Vernon S E, Acar B C, Pham S M, Fertel D. Acanthamoeba infection in lung transplantation: report of a case and review of the literature. Transpl Infect Dis 2005; 7: 154-7.

19.- Panjwani N, Zhao Z, Baum J, Hazlett L D, Yang Z. Acanthamoebae bind to rabbit corneal epithelium in vitro. Invest Ophthalmol Vis Sci 1997; 38: 1858-64.

20.- Garate M, Marchant J, Cubillos I, Cao Z, Khan N A, Panjwani N. In vitro pathogenicity of Acanthamoeba is associated with the expression of the mannose-binding protein. Invest Ophthalmol Vis Sci 2006; 47: 1056-62.

21.- Garate M, Cao Z, Bateman E, Panjwani N. Cloning and characterization of a novel mannose-binding protein of Acanthamoeba. J Biol Chem 2004; 279: 29849-56.

22.- Siddiqui R, Emes R, Elsheikha H, Khan N A Area 51: How do Acanthamoeba invade the central nervous system? Trends Parasitol 2011; 27: $185-9$

23.- Siddiqui R, Khan N A. Biology and pathogenesis of Acanthamoeba. Parasit Vectors 2012; 5: 6.

24.- Khan N A. Acanthamoeba: biology and increasing importance in human health. FEMS Microbiol Rev 2006; 30: 564-95.

25.- Khan N A. Pathogenicity, morphology, and differentiation of Acanthamoeba. Curr Microbiol 2001; 43: 391-5.

26.- Ferrante A, Rowan-Kelly B. Activation of the alternative pathway of complement by Acanthamoeba culbertsoni. Clin Exp Immunol 1983; 54: 477-85.
27.- Pumidonming W, Walochnik J, Dauber E, Petry F. Binding to complement factors and activation of the alternative pathway by Acanthamoeba. Immunobiology 2011; 216 : 225-33.

28.- Alizadeh H, Neelam S, Niederkorn J Y. Role of activated macrophages in Acanthamoeba keratitis. J Parasitol 2007; 93: 1114-20.

29.- Hurt M, Proy V, Niederkorn J Y, Alizadeh H. The interaction of Acanthamoeba castellanii cysts with macrophages and neutrophils. J Parasitol 2003; 89: 565-72.

30.- Marciano-Cabral F, Toney D M. The interaction of Acanthamoeba spp. with activated macrophages and with macrophage cell lines. J Eukaryot Microbiol 1998; 45: 452-8.

31.- Ren M Y, Wu X Y. Toll-like receptor 4 signalling pathway activation in a rat model of Acanthamoeba keratitis. Parasite Immunol 2011; 33: 25-33.

32.- Clarke D W, Niederkorn J Y. The immunobiology of Acanthamoeba keratitis. Microbes Infect 2006; 8: 1400-5.

33.- Niederkorn J Y, Alizadeh H, Leher H F, McCulley J P. The immunobiology of Acanthamoeba keratitis. Springer Semin Immunopathol 1999; 21: 147-60.

34.- McClellan K, Howard K, Mayhew E, Niederkorn J, Alizadeh H. Adaptive immune responses to Acanthamoeba cysts. Exp Eye Res 2002; 75: 285-93.

35.- Leher H F, Alizadeh H, Taylor W M, Shea A S, Silvany R S, Van Klink F, et al. Role of mucosa IgA in the resistance to Acanthamoeba keratitis Invest Ophthalmol Vis Sci 1998; 39: 2666-73.

36.- Marciano-Cabral F, Puffenbarger R, Cabral G A. The increasing importance of Acanthamoeba infections. J Eukaryot Microbiol 2000; 47: 2936

37.- Harrison J L, Ferreira G A, Raborn E S, Lafrenaye A D, Marciano-Cabral F, Cabral G A. Acanthamoeba culbertsoni elicits soluble factors that exert anti-microglial cell activity. Infect Immun 2010; 78: 4001-11.

38.- Hurt M, Niederkorn J, Alizadeh H. Effects of mannose on Acanthamoeba castellanii proliferation and cytolytic ability to corneal epithelial cells. Invest Ophthalmol Vis Sci 2003; 44: 3424-31

39.- Anderson I J, Watkins R F, Samuelson J, Spencer D F, Majoros W H, Gray M W, et al. Gene discovery in the Acanthamoeba castellanii genome. Protist 2005; 156: 203-14.

40.- Schuster F L. Cultivation of pathogenic and opportunistic free-living amebas. Clin Microbiol Rev 2002; 15: 342-54.

41.- Parmar D N, Awwad S T, Petroll W M, Bowman R W, McCulley J P, Cavanagh H D. Tandem scanning confocal corneal microscopy in the diagnosis of suspected Acanthamoeba keratitis. Ophthalmology 2006; 113: 538-47.

42.- Dart J K, Saw V P, Kilvington S. Acanthamoeba keratitis: diagnosis and treatment update 2009. Am J Ophthalmol 2009; 148: 487-99 e2.

43.- Lai S, Asgari M, Henney H R, Jr. Nonradioactive DNA probe and polymerase chain reaction procedures for the specific detection of Acanthamoeba. Mol Cell Probes 1994; 8: 81-9.

44.- Schuster F L, Visvesvara G S. Free-living amoebae as opportunistic and non-opportunistic pathogens of humans and animals. Int $\mathrm{J}$ Parasitol 2004; 34: 1001-27.

45.- Kong H H, Shin J Y, Yu H S, Kim J, Hahn T W, Hahn Y H, et al. Mitochondrial DNA restriction fragment length polymorphism (RFLP) and $18 \mathrm{~S}$ small-subunit ribosomal DNA PCR-RFLP analyses of Acanthamoeba isolated from contact lens storage cases of residents in southwestern Korea. J Clin Microbiol 2002; 40: 1199-206.

46.- Schuster F L, Visvesvara G S. Amebae and ciliated protozoa as causal agents of waterborne zoonotic disease. Vet Parasitol 2004; 126: 91-120.

47.- Ayers K M, Billups L H, Garner F M. Acanthamoebiasis in a dog. Vet Pathol 1972; 9: 221-6

48.- Kadlec V. The occurrence of amphizoic Amebae in domestic animals. J Protozool 1978; 25: 2357.

49.- Schuster F L, Visvesvara G S. Opportunistic amoebae: challenges in prophylaxis and treatment. Drug Resist Updat 2004; 7: 41-51.

50.- Singhal T, Bajpai A, Kalra V, Kabra S K, Samantaray J C, Satpathy G, et al. Successful treatment of Acanthamoeba meningitis with combination oral antimicrobials. Pediatr Infect Dis J 2001; 20: 623-7.

51.- Karande S C, Lahiri K R, Sheth S S, Nadkarni U S, Jain M K, Shah M D. Acanthamoeba meningoencephalitis complicating pyogenic meningitis. Indian Pediatr 1991; 28: 794-7.

52.- Slater C A, Sickel J Z, Visvesvara G S, Pabico R C, Gaspari A A. Brief report: successful treatment of disseminated Acanthamoeba infection in an immunocompromised patient. N Engl J Med 1994; 331: 85-7.

53.- Seijo Martínez M, González-Mediero G, Santiago P, Rodríguez De Lope A, Diz J, Conde $\mathrm{C}$, et al. Granulomatous amebic encephalitis in a patient with AIDS: isolation of Acanthamoeba sp. Group II from brain tissue and successful treatment with sulfadiazine and fluconazole. J Clin Microbiol 2000; 38: 3892-5.

54.- Bloch K C, Schuster F L. Inability to make a premortem diagnosis of Acanthamoeba species infection in a patient with fatal granulomatous amebic encephalitis. J Clin Microbiol 2005; 43: 3003-6.

55.- Seal D V. Acanthamoeba keratitis updateincidence, molecular epidemiology and new drugs for treatment. Eye (Lond) 2003; 17: 893 905 
56.- Elder M J, Kilvington S, Dart J K. A clinicopathologic study of in vitro sensitivity testing and Acanthamoeba keratitis. Invest Ophthalmol Vis Sci 1994; 35: 1059-64.

57.- Ferrari G, Matuska S, Rama P. Doublebiguanide therapy for resistant Acanthamoeba keratitis. Case Report Ophthalmol 2011; 2: 338-42.

58.- Polat Z A, Vural A. Effect of combined chlorhexidine gluconate and neosporin on experimental keratitis with two pathogenic strains of Acanthamoeba. Parasitol Res 2012; 110 (5): 1945-50.

59.- Azuara-Blanco A, Sadiq A S, Hussain M, Lloyd J H, Dua H S. Successful medical treatment of Acanthamoeba keratitis. Int Ophthalmol 1997; 21: 223-7.

60.- Oliva S, Jantz M, Tiernan R, Cook D L, Judson M A. Successful treatment of widely disseminated acanthamoebiasis. South Med J
1999; 92: 55-7.

61.- Kosrirukvongs P, Wanachiwanawin D, Visvesvara G S. Treatment of Acanthamoeba keratitis with chlorhexidine. Ophthalmology 1999; 106: 798-802.

62.- Seal D, Hay J, Kirkness C, Morrell A, Booth A, Tullo A, et al. Successful medical therapy of Acanthamoeba keratitis with topical chlorhexidine and propamidine. Eye (Lond) 1996; 10 ( Pt 4): 413-21. 